\title{
ESTRATÉGIAS DE ENSINO E APRENDIZAGEM DA RESSUSCITAÇÃO CARDIOPULMONAR NEONATAL PARA ENFERMAGEM: REVISÃO INTEGRATIVA DA LITERATURA
}

\section{TEACHING AND LEARNING STRATEGIES FOR NEONATAL CARDIOPULMONARY RESUSCITATION FOR NURSING: INTEGRATIVE LITERATURE REVIEW}

\section{ESTRATEGIAS DE ENSEÑANZA Y APRENDIZAJE PARA REANIMACIÓN CARDIOPULMONAR NEONATAL PARA ENFERMERÍA: REVISIÓN INTEGRATIVA DE LITERATURA}

\author{
Jordana Luiza Gouvêa de Oliveira ${ }^{1}$, Juliana da Silva Garcia Nascimento ${ }^{2}$, Maria Célia Barcellos Dalri ${ }^{3}$, Gabriel Segura Torres ${ }^{4}$
}

\section{RESUMO}

Objetivo: Analisar evidências científicas sobre as estratégias de ensino e aprendizagem quanto à ressuscitação cardiopulmonar neonatal utilizadas na atualidade para a enfermagem. Método: Revisão integrativa realizada, nas bases de dados National Library of Medicine, no National Institutes of Health, na Cumulative Index to Nursing and Allied Health Literature, na Latin-American and Caribbean System on Health Sciences Information, Embase, por meio do aplicativo Rayyan. Resultados: Identificaram-se 244 estudos e selecionaram-se 21. Elaboraram-se as categorias: principais estratégias de ensino e aprendizagem sobre ressuscitação cardiopulmonar neonatal; efetividade das estratégias de ensino e aprendizagem e lacunas no processo de ensino e aprendizagem. As principais estratégias foram: programa Helping Babies Breathe; simulação em laboratório, simulação in situ; e associação de estratégias. Todas se apresentaram efetivas, para o processo de ensino e aprendizagem, e a lacuna identificada foi a dificuldade em reter o conhecimento adquirido. Conclusão: As estratégias são na maioria inovadoras e efetivas para o processo de ensino e aprendizagem da ressuscitação cardiopulmonar neonatal.

Descritores: Reanimação Cardiopulmonar. Recém-nascido. Educação em Enfermagem.

\section{ABSTRACT}

Objective: To analyze scientific evidence on teaching and learning strategies regarding neonatal cardiopulmonary resuscitation currently used for nursing. Method: Integrative review conducted in the National Library of Medicine databases, the National Institutes of Health, the Cumulative Index to Nursing and Allied Health Literature, the Latin American and Caribbean System on Health Sciences Information and Embase, using Rayyan applications. Results: a total of 244 studies were identified and 21 were selected. The following categories were elaborated: main teaching and learning strategies on neonatal cardiopulmonary resuscitation; effectiveness of teaching and learning strategies; and gaps in the teaching and learning process. The main strategies were: Helping Babies Breathe program; laboratory simulation, in situ simulation; and strategy association. All categories were effective for the teaching and learning process and the identified gap was the difficulty in retaining the acquired knowledge. Conclusion: The applied strategies are mostly innovative and effective for the teaching and learning process of neonatal cardiopulmonary resuscitation.

Descriptors: Cardiopulmonary Resuscitation; Infant, Newborn; Nursing Education.

\section{RESUMEN}

Objetivo: analizar la evidencia científica sobre estrategias de enseñanza y aprendizaje con respecto a la reanimación cardiopulmonar neonatal que se utiliza actualmente en enfermería. Método: revisión integradora realizada en las bases de datos de la Biblioteca Nacional de Medicina, los Institutos Nacionales de Salud, el Índice Acumulativo de Literatura de Enfermería y Salud Aliada, el Sistema Latinoamericano y del Caribe de Información en Ciencias de la Salud, Embase, a través de la aplicación Rayyan. Resultados: se identificaron 244 estudios y se seleccionaron 21. Se elaboraron las siguientes categorías: principales estrategias de enseñanza y aprendizaje sobre reanimación cardiopulmonar neonatal; efectividad de las estrategias de enseñanza y aprendizaje; y lagunas en el proceso de enseñanza y aprendizaje. Las estrategias principales fueron: programa Ayudando a los Bebés a Respirar; simulación de laboratorio, simulación in situ; y asociación de estrategias. Todos fueron efectivos para el proceso de enseñanza y aprendizaje y la brecha identificada fue la dificultad para retener el conocimiento adquirido. Conclusión: las estrategias son, en su mayoría, innovadoras y efectivas para el proceso de enseñanza y aprendizaje de la reanimación cardiopulmonar neonatal. Descriptores: Reanimación Cardiopulmonar; Recién Nacido; Educación en Enfermería.

${ }^{1}$ Enfermeira graduada pela Escola de Enfermagem de Ribeirão Preto da Universidade de São Paulo. Mestranda do Programa de Enfermagem Fundamental da Escola de Enfermagem de Ribeirão Preto, Universidade de São Paulo. ${ }^{2}$ Mestre em enfermagem. Docente no curso de graduação em enfermagem da Universidade de Uberaba, Minas Gerais. ${ }^{3}$ Professora Associada do Departamento de Enfermagem Geral e Especializada da Escola de Enfermagem de Ribeirão Preto, Universidade de São Paulo. ${ }^{4}$ Médico formado pela Universidad de Iberoamérica, Sán José, Costa Rica.

Como citar este artigo:

Oliveira JLG, Nascimento JSG, Dalri MCB, et al. Estratégias de ensino e aprendizagem da ressuscitação cardiopulmonar neonatal para enfermagem: revisão integrativa da literatura. Revista de Enfermagem do Centro-Oeste Mineiro. 2019;9:e3572.

[Access ; Disponível em: DOI: http://dx.doi.org/10.19175/recom.v9i0.3572 


\section{INTRODUÇÃO}

No cenário global, três milhões de recémnascidos morrem por ano e $99 \%$ desses óbitos ocorrem em países em desenvolvimento e com poucos recursos ${ }^{(1)}$. No Brasil, a cada ano, ocorrem três milhões de nascimentos e um número de óbitos anual de, aproximadamente, 25.608 neonatos, caracterizados, em sua maioria, por causas preveníveis ${ }^{(2)}$.

Neste cenário, a American Heart Association (AHA), considerada a maior organização americana voluntária, dedicada ao combate das doenças cardiovasculares e utilizada como principal referencial teórico, para a ressuscitação cardiopulmonar neonatal (RCP-N), endossa a necessidade de aperfeiçoar este ensino clínico para aumento da sobrevida desta população ${ }^{(1)}$.

Para o cumprimento deste objetivo, a literatura aponta que é imprescindível o investimento internacional e nacional de instituições de ensino e saúde, quanto ao processo de aprendizagem da RCP-N e que o atendimento eficiente e seguro de uma PCR-N inicia-se na prática educativa. Porém identificase, ainda, uma taxa de não aderência aos protocolos, para esse atendimento de $16 \%$ a $55 \%$, nos ambientes de atendimento à saúde e uma adesão exacerbada a estratégias de aprendizagem exclusivamente tradicionais, o que permite enfatizar a necessidade de motivação, por meio de estratégias pedagógicas inovadoras, que desenvolvam competência clínica e favoreçam o seguimento dos guidelines ${ }^{(1-2)}$.

Apesar de constatar que o enfermeiro é, geralmente, o primeiro profissional a identificar uma PCR-N $\mathrm{N}^{(1,3)}$ e a ênfase crescente, nas últimas décadas, sobre a necessidade de se otimizar as estratégias de ensino e aprendizagem, quanto à essa temática, para a enfermagem, as possibilidades pedagógicas, utilizadas por docentes, instituições de ensino e saúde, nem sempre são acompanhadas de resultados estatisticamente significantes para a efetividade da RCP-N $\mathrm{N}^{(3)}$. Soma-se a esse cenário a incipiência de estudos experimentais randomizados, bem delineados, que possuem a intencionalidade de comparar a efetividade de estratégias e mecanismos de ensino da RCP-N, visando subsidiar a prática baseada em evidências e, também, pesquisas de revisão de literatura que se aprofundem sobre a temática ${ }^{(3)}$.

Esta fragilidade quanto à exploração do conhecimento existente, associada à importância de se identificar e compreender as formas adotadas, para mediar a aquisição de habilidade cognitiva, motora e afetiva de profissionais e estudantes de enfermagem para RCP-N, instigase a realização de uma revisão integrativa da literatura (RIL), que se proponha a realizar um mapeamento das atuais estratégias de ensino neste âmbito e contribuir, para a ciência da enfermagem e a assistência neonatal, oferecendo um sólido arcabouço pedagógico de determinação das melhores práticas educacionais que impactem positivamente em uma RCP-N de excelência. Esta pesquisa objetivou, portanto analisar as evidências científicas sobre estratégias de ensino e aprendizagem quanto à RCP-N utilizadas na atualidade para a enfermagem.

\section{MÉTODO}

Trata-se de RIL sobre estratégias de ensino e aprendizagem de RCP-N para enfermagem adotadas na atualidade. Este tipo de pesquisa possibilita a análise de estudos relevantes, embasamento para a tomada de decisões, aprimoramento para a prática profissional, bem como o reconhecimento e exploração do tema proposto $^{(4)}$.

Para a elaboração da presente revisão, foram cumpridas as etapas: determinação da questão norteadora, definição da amostragem, com os respectivos critérios de inclusão e exclusão, categorização dos estudos, análise dos estudos incluídos, interpretação dos resultados e reprodução da revisão ${ }^{(4)}$.

A questão norteadora de pesquisa, elaborada por meio da estratégia PICO, foi embasada na identificação do acrônimo "P", referente à população e configurada nesta revisão por profissionais e estudantes em enfermagem. O acrônimo "I" indica a intervenção proposta, neste contexto, representada pela aplicação de estratégias de ensino e aprendizagem para a RCP-N, e o acrônimo "O" considera o desfecho pretendido, neste caso, determinado pela aprendizagem da RCP- $\mathrm{N}^{(4)}$. Formulou-se, desta forma, a pergunta: quais são as evidências científicas atuais existentes na literatura sobre as estratégias de ensino e aprendizagem da RCP-N para a enfermagem?

A seleção dos estudos ocorreu de abril a junho de 2019, por meio de três fases. A fase 1 abordou a busca dos estudos nas bases de dados PubMed $^{\circledR}$, EMBASE, Cumulative Index to Nursing and Allied Health Literature (CINAHL) e LILACS. Definiu-se como critérios de inclusão: artigo 
primário, publicado nos últimos cinco anos, visto que a publicação da última diretriz sobre o tema data de $2015^{(1)}$, em inglês, português e espanhol, relacionada com o ensino da RCP-N para profissionais e estudantes de enfermagem. Excluíram-se estudos relacionados à RCP, em prematuros extremos, cuidados paliativos, cuidados pediátricos, emergências obstétricas e RCP-N para leigos.

Na base de dados PubMed ${ }^{\circledR}$, os descritores foram identificados no Medical Subjects Headings (MeSH), tendo sido determinada a estratégia de busca: Resuscitation OR "Cardio Pulmonary Resuscitation" OR "Cardiac Life Support" OR cpr AND "Infant, Newborn" OR newborn* OR Neonat* AND education* $O R$ learning $O R$ teaching AND nurs*. Na base de dados EMBASE, a busca se deu por palavras, em todos os campos, caracterizando a estratégia: "resuscitation"/exp $O R$ resuscitation $O R$ "cardiac life support" $O R \mathrm{cpr}$ AND newborn* $O R$ neonat* AND education* $O R$ "learning"/exp OR learning $O R$ "teaching"/exp $O R$ teaching AND nurs*. Já, na CINAHL, os descritores foram identificados em títulos e realizou-se a estratégia: Resuscitation $O R$ "Cardiac Life Support" OR cpr AND "Infant, Newborn" OR newborn* OR Neonat* AND education* OR learning OR teaching AND nurs*. Na LILACS, a busca foi por palavras por meio da estratégia: (reanimacao $O R$ Ressuscitação) and (RecémNascid\$) $O R$ (recém $A N D$ nascid\$) $O R$ Neonat\$ and (educação $O R$ ensino $O R$ gradua $\$$ ) $A N D$ (enfermagem $O R$ enfermeir\$).

A fase dois abrangeu a avaliação de títulos e resumos dos estudos, por dois pesquisadores independentes.
Os 244 artigos foram exportados para a plataforma Rayyan, tecnologia que agiliza a triagem, com alto nível de exatidão para a seleção dos estudos ${ }^{(5)}$. Os 14 estudos que apresentaram divergência foram direcionados a um terceiro pesquisador. A fase três correspondeu à leitura na íntegra dos artigos e à decisão da amostra final. Utilizou-se um instrumento já validado, para coleta e organização dos achados ${ }^{(6)}$, que abordou: número do estudo, título, periódico, ano da publicação, objetivos, método, resultados e conclusão, classificando-se, também, o nível de evidência dos estudos. Por fim, os resultados foram analisados e categorizados ${ }^{(4)}$, em três principais temas, considerando as informações e descrições da amostra selecionada mais frequentes e relevantes ao tema, que se destacaram quanto à intencionalidade da questão norteadora do presente estudo, a saber: (1) as principais estratégias de ensino e aprendizagem sobre RCP$\mathrm{N}$, que explanam o cenário do que vem sendo adotado na atualidade para este fim; (2) a efetividade das estratégias de ensino e aprendizagem sobre RCP-N, possibilitando identificar quais mecanismos pedagógicos são mais eficientes para conduzir a escolha de profissionais e docentes em enfermagem e (3) as lacunas do processo de ensino e aprendizagem quanto à estratégia utilizada para RCP-N, para reflexão sobre as fragilidades e dificuldades envoltas neste contexto.

A seleção dos estudos é demonstrada na Figura 1, de acordo com as recomendações Preferred Reporting Items for Systematic Reviews and Meta-Analyses (PRISMA) ${ }^{(7)}$. 
Figura 1 - Fluxograma do processo de identificação, seleção e inclusão dos estudos elaborado, a partir da recomendação PRISMA. Ribeirão Preto (SP), Brasil, 2019. CINAHL: Cumulative Index to Nursing and Allied Health Literature.

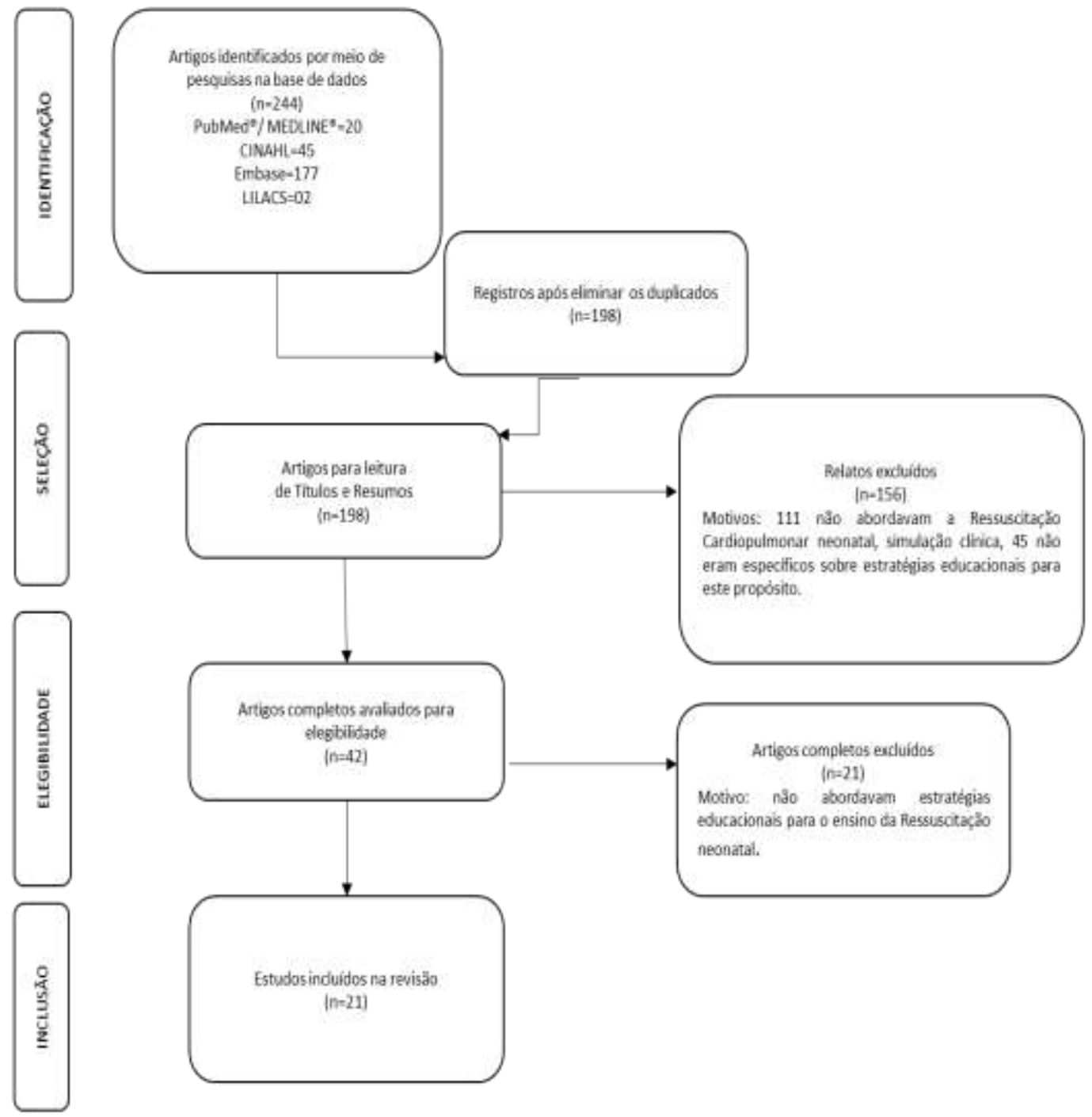

\section{RESULTADOS E DISCUSSÃO}

Identificaram-se 21 estudos que compuseram a mostra final desta pesquisa, os quais se encontram caracterizados no Quadro 1, a seguir:

Figura 2- caracterização dos estudos acerca de sua intencionalidade, delineamento metodológico, principais resultados e desfechos.

\begin{tabular}{|c|c|c|c|c|c|}
\hline $\begin{array}{c}\text { Autor/ } \\
\text { ano }\end{array}$ & Título do estudo & Objetivo & Método & Resultados/conclusão & NE \\
\hline $\begin{array}{l}\text { (Brito et } \\
\text { al, 2017) }^{(8)}\end{array}$ & $\begin{array}{l}\text { Neonatal Resuscitation Program } \\
\text { Rolling Refresher: Maintaining Chest } \\
\text { Compression Proficiency Through the } \\
\text { Use of Simulation-Based Education }\end{array}$ & $\begin{array}{c}\text { Analisar a } \\
\text { simulação in } \\
\text { situ }\end{array}$ & $\begin{array}{l}\text { Estudo } \\
\text { experimental, } \\
\text { randomizado com } \\
25 \text { enfermeiros. }\end{array}$ & $\begin{array}{l}\text { Os escores, para } \\
\text { confiança e habilidades } \\
\text { em RCP-N, foram } \\
\text { estatisticamente } \\
\text { significativos. }\end{array}$ & 2 \\
\hline
\end{tabular}

"continua na página seguinte" 


\begin{tabular}{|c|c|c|c|c|c|}
\hline $\begin{array}{l}\text { Autor/ } \\
\text { ano }\end{array}$ & Título do estudo & Objetivo & Método & Resultados/conclusão & NE \\
\hline $\begin{array}{l}\text { (Kasinea et } \\
\text { al, 2018) }^{(1)}\end{array}$ & $\begin{array}{l}\text { Translating continuing professional } \\
\text { development education to nursing } \\
\text { practice in Rwanda: Enhancing } \\
\text { maternal and newborn health }\end{array}$ & $\begin{array}{l}\text { Explorar o } \\
\text { curso HBB }\end{array}$ & $\begin{array}{l}\text { Estudo descritivo, } \\
\text { qualitativo, } \\
\text { realizado em } \\
\text { Ruanda. }\end{array}$ & $\begin{array}{l}\text { A confiança das } \\
\text { enfermeiras sobre a RCP- } \\
\mathrm{N} \text { foi estatisticamente } \\
\text { significativa. }\end{array}$ & 6 \\
\hline $\begin{array}{l}\text { (Malmström } \\
\text { et } a, 2017)^{(2)}\end{array}$ & $\begin{array}{l}\text { Simulation-based team training } \\
\text { improved the self-assessed ability of } \\
\text { physicians, nurses and midwives to } \\
\text { perform neonatal resuscitation }\end{array}$ & $\begin{array}{l}\text { Avaliar a } \\
\text { simulação em } \\
\text { equipe para } \\
\text { RCPN }\end{array}$ & $\begin{array}{l}\text { Estudo quasi- } \\
\text { experimental, na } \\
\text { Suécia, que avaliou } \\
\text { um treinamento em } \\
\text { equipe baseado em } \\
\text { simulação. }\end{array}$ & $\begin{array}{l}\text { Foram avaliados: } \\
\text { comunicação, liderança, } \\
\text { confiança e habilidades } \\
\text { técnicas e identificou-se } \\
\text { melhora em todos. }\end{array}$ & 3 \\
\hline $\begin{array}{l}\text { (Perlman et } \\
\mathrm{al}, 2016)^{(3)}\end{array}$ & $\begin{array}{l}\text { Designing and Implementing the } \\
\text { Helping Babies Breathe Program in } \\
\text { Tanzania }\end{array}$ & $\begin{array}{l}\text { Descrever o } \\
\text { HBB }\end{array}$ & $\begin{array}{l}\text { Estudo descritivo, } \\
\text { sobre a abordagem } \\
\text { do programa HBB. }\end{array}$ & $\begin{array}{l}\text { O programa ocasionou } \\
\text { redução da mortalidade } \\
\text { neonatal. }\end{array}$ & 6 \\
\hline $\begin{array}{l}\text { (Rovamo et } \\
\text { al, 2015) }^{(9)}\end{array}$ & $\begin{array}{l}\text { Effect of a simulation- based } \\
\text { workshop on multidisplinary } \\
\text { teamwork of newborn emergencies: } \\
\text { an intervention study }\end{array}$ & $\begin{array}{c}\text { Avaliar o } \\
\text { impacto da } \\
\text { simulação de } \\
\text { RCP-N }\end{array}$ & $\begin{array}{l}\text { Estudo } \\
\text { experimental, } \\
\text { randomizado, } \\
\text { realizado com } 99 \\
\text { indivíduos } \\
\text { Finlândia. }\end{array}$ & $\begin{array}{l}\text { Não ocorreram } \\
\text { diferenças } \\
\text { estatisticamente } \\
\text { significativas entre os } \\
\text { grupos. }\end{array}$ & 2 \\
\hline $\begin{array}{l}\text { (Saeidi et al, } \\
2017)^{(10)}\end{array}$ & $\begin{array}{l}\text { Comparison of Effect of Simulation- } \\
\text { based Neonatal Resuscitation } \\
\text { Education and Traditional Education } \\
\text { on Knowledge of Nursing Students }\end{array}$ & $\begin{array}{l}\text { Comparar a } \\
\text { efetividade da } \\
\text { simulação RCP- } \\
\text { N }\end{array}$ & $\begin{array}{l}\text { Estudo experimental } \\
\text { realizado na } \\
\text { Faculdade de } \\
\text { Enfermagem, no Irã, } \\
\text { com } 80 \text { enfermeiros. }\end{array}$ & $\begin{array}{l}\text { A pontuação média dos } \\
\text { alunos submetidos à } \\
\text { simulação roi } \\
\text { significativamente maior. }\end{array}$ & 2 \\
\hline $\begin{array}{l}\text { (Shee et a, } \\
2018)^{(11)}\end{array}$ & $\begin{array}{l}\text { Impact of the neoresus training on } \\
\text { nonpediatric- trained medical and } \\
\text { nursing staff in the emergency } \\
\text { department: Experience from two } \\
\text { sites in regional Australia }\end{array}$ & $\begin{array}{l}\text { Determinar se } \\
\text { o NeoResus é } \\
\text { efetivo para } \\
\text { RCPN }\end{array}$ & $\begin{array}{lr}\text { Estudo descritivo, } \\
\text { realizado com } 45 \\
\text { profissionais } & \text { de } \\
\text { hospitais } & \\
\text { australianos. }\end{array}$ & $\begin{array}{l}\text { Os cenários de RCP-N } \\
\text { melhoraram a confiança. }\end{array}$ & 6 \\
\hline $\begin{array}{l}\text { (Vail et al, } \\
2017)^{(12)}\end{array}$ & $\begin{array}{l}\text { Care of the mother-infant dyad: a } \\
\text { novel approach to conducting and } \\
\text { evaluating neonatal resuscitation } \\
\text { simulation training in Bihar, India }\end{array}$ & $\begin{array}{l}\text { Avaliar o } \\
\text { impacto do } \\
\text { programa } \\
\text { "PRONTO" }\end{array}$ & $\begin{array}{lr}\text { Estudo transversal, } \\
\text { quantitativo } & \text { que } \\
\text { avaliou } & 658 \\
\text { enfermeiros } & \text { de } \\
\text { Bihar, Índia. } & \end{array}$ & $\begin{array}{l}\text { O treinamento PRONTO } \\
\text { foi capaz de desenvolver } \\
\text { as habilidades quanto à } \\
\text { RCP neonatal. }\end{array}$ & 6 \\
\hline $\begin{array}{l}\text { (Wilson et } \\
\text { al, 2017) }^{(13)}\end{array}$ & 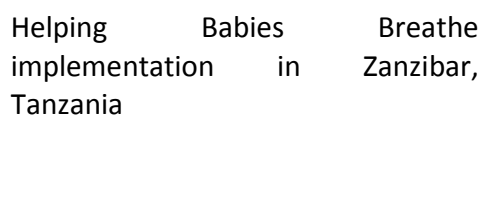 & $\begin{array}{c}\text { Avaliar a } \\
\text { eficácia do } \\
\text { programa HBB }\end{array}$ & $\begin{array}{l}\text { Métodos mistos, } \\
\text { realizado na } \\
\text { Tanzânia, com } 33 \\
\text { participantes de } 24 \\
\text { unidades de saúde. }\end{array}$ & $\begin{array}{lr}\text { Os escores de } \\
\text { conhecimento } & \text { e } \\
\text { habilidades para } & \text { RCP-N } \\
\text { melhoraram } & \\
\text { significativamente. }\end{array}$ & 6 \\
\hline $\begin{array}{l}\text { Reed et al, } \\
2017)^{(14)}\end{array}$ & $\begin{array}{l}\text { Interdisciplinary onsite team-based } \\
\text { simulation training in the neonatal } \\
\text { intensive care unit. }\end{array}$ & $\begin{array}{c}\text { Relatar sobre } \\
\text { uma RCP-N }\end{array}$ & $\begin{array}{ll}\text { Relato } & \text { de } \\
\text { experiência, } & \\
\text { realizado em UTI } \\
\text { neonatal, com } 500 \\
\text { funcionários. }\end{array}$ & $\begin{array}{l}\text { Incluiu-se o acionamento } \\
\text { da equipe do código azul. } \\
\text { O treinamento } \\
\text { demonstrou-se } \\
\text { apropriado. }\end{array}$ & 6 \\
\hline $\begin{array}{l}\text { (Umar et al, } \\
2018)^{(15)}\end{array}$ & $\begin{array}{l}\text { Evaluation of the Cognitive Effect of } \\
\text { Newborn Resuscitation Training on } \\
\text { Health-care Workers in Selected } \\
\text { States in Northern Nigeria }\end{array}$ & $\begin{array}{l}\text { Avaliar o } \\
\text { impacto de um } \\
\text { curso de RCP-N }\end{array}$ & $\begin{array}{l}\text { Estudo quasi- } \\
\text { experimental, com } \\
293 \text { profissionais de } \\
\text { saúde que } \\
\text { participaram de } \\
\text { treinamento sobre a } \\
\text { RCP-N na Nigéria. }\end{array}$ & $\begin{array}{l}\text { Apresentaram melhorias } \\
\text { nos escores no pós-teste, } \\
\text { concluindo que } \\
\text { treinamento de RCP } \\
\text { melhorou } \\
\text { conhecimento. }\end{array}$ & 3 \\
\hline
\end{tabular}


"continua na página seguinte"

\begin{tabular}{|c|c|c|c|c|c|}
\hline $\begin{array}{l}\text { Autor/ } \\
\text { ano }\end{array}$ & Título do estudo & Objetivo & Método & Resultados/conclusão & NE \\
\hline $\begin{array}{l}\text { (Charafeddi } \\
\text { ne et al, } \\
2016)^{(16)}\end{array}$ & $\begin{array}{l}\text { Strategic assessment of } \\
\text { implementation of neonatal } \\
\text { resuscitation training at a national } \\
\text { level }\end{array}$ & $\begin{array}{l}\text { Relatar sobre } \\
\text { um programa } \\
\text { de RCP }\end{array}$ & $\begin{array}{l}\text { Estudo descritivo } \\
\text { sobre um programa } \\
\text { de treinamento em } \\
\text { RCP em } 32 \text { hospitais } \\
\text { de Beirute. }\end{array}$ & $\begin{array}{l}\text { Os escores foram } \\
\text { significativamente } \\
\text { maiores quanto ao } \\
\text { conhecimento após o } \\
\text { treinamento. }\end{array}$ & 6 \\
\hline $\begin{array}{l}\text { (Cordova et } \\
\text { al, 2018) }^{(17)}\end{array}$ & $\begin{array}{l}\text { Effect of low-cost interventions on } \\
\text { the retention of knowledge and } \\
\text { skills following Helping Babies } \\
\text { Breathe training }\end{array}$ & $\begin{array}{l}\text { Avaliar o } \\
\text { impacto do } \\
\text { programa HBB } \\
\text { para RCP-N }\end{array}$ & $\begin{array}{l}\text { Estudo quasi- } \\
\text { experimental, que } \\
\text { utilizou o HBB em } \\
\text { Cajamarca, Peru. }\end{array}$ & $\begin{array}{l}\text { Não se identificaram } \\
\text { diferenças significativas } \\
\text { entre as avaliações } \\
\text { imediatas e após seis } \\
\text { meses. }\end{array}$ & 3 \\
\hline $\begin{array}{l}\text { (Ashish et al, } \\
2017)^{(18)}\end{array}$ & $\begin{array}{l}\text { Evaluation of Helping Babies } \\
\text { Breathe Quality Improvement Cycle } \\
\text { (HBB-QIC) on retention of neonatal } \\
\text { resuscitation skills six months after } \\
\text { training in Nepal }\end{array}$ & $\begin{array}{c}\text { Avaliar o } \\
\text { impacto do } \\
\text { HBB para RCP- } \\
\text { N }\end{array}$ & $\begin{array}{l}\text { Estudo quasi- } \\
\text { experimental, que } \\
\text { avaliou o impacto } \\
\text { do HBB. }\end{array}$ & $\begin{array}{l}\text { Escores de conhecimento } \\
\text { e habilidades foram } \\
\text { maiores após o } \\
\text { treinamento. }\end{array}$ & 3 \\
\hline $\begin{array}{l}\text { (Vail et al, } \\
2018)^{(19)}\end{array}$ & $\begin{array}{l}\text { The power of practice: simulation } \\
\text { training improving the quality of } \\
\text { neonatal resuscitation skills in Bihar, } \\
\text { India }\end{array}$ & $\begin{array}{l}\text { Avaliar o } \\
\text { treinamento } \\
\text { "PRONTO" }\end{array}$ & $\begin{array}{l}\text { Estudo de método } \\
\text { misto que avaliou o } \\
\text { impacto de um } \\
\text { treinamento sobre } \\
\text { RCP-N, para } 1.342 \\
\text { enfermeiros, em } \\
\text { Bihar, Índia. }\end{array}$ & $\begin{array}{l}\text { O treinamento proposto } \\
\text { teve impacto positivo no } \\
\text { desenvolvimento e na } \\
\text { qualidade } \\
\text { habilidades de RCP. }\end{array}$ & 6 \\
\hline $\begin{array}{l}\text { (Bull et al, } \\
2015)^{(20)}\end{array}$ & $\begin{array}{l}\text { Midwifery students receiving the } \\
\text { newborn at birth: A pilot study of } \\
\text { the impact of structured training in } \\
\text { neonatal resuscitation }\end{array}$ & $\begin{array}{l}\text { Relatar um } \\
\text { treinamento } \\
\text { sobre RCP-N }\end{array}$ & $\begin{array}{l}\text { Estudo quasi- } \\
\text { experimental, } \\
\text { realizado com um } \\
\text { único grupo, } \\
\text { formado por seis } \\
\text { estudantes de } \\
\text { enfermagem, na } \\
\text { Austrália. }\end{array}$ & $\begin{array}{l}\text { O conhecimento do } \\
\text { grupo aumentou. Os } \\
\text { alunos se sentiram mais } \\
\text { preparados para a RCP- } \\
\mathrm{N} \text {. }\end{array}$ & 3 \\
\hline $\begin{array}{l}\text { (Carolan- } \\
\text { Olah et al, } \\
2016)^{(21)}\end{array}$ & $\begin{array}{l}\text { Development and evaluation of a } \\
\text { simulation exercise to prepare } \\
\text { midwifery students for neonatal } \\
\text { resuscitation }\end{array}$ & $\begin{array}{l}\text { Avaliar uma } \\
\text { simulação de } \\
\text { RCP-N }\end{array}$ & $\begin{array}{lr}\text { Pesquisa descritiva } \\
\text { sobre } \\
\text { desenvolvimento de } \\
\text { uma simulação de } \\
\text { RCP-N, para } 36 \\
\text { estudantes } \\
\text { enfermagem. }\end{array}$ & $\begin{array}{l}\text { A simulação resultou na } \\
\text { melhora da confiança do } \\
\text { aluno e no aumento do } \\
\text { conhecimento em RCP-N. }\end{array}$ & 6 \\
\hline $\begin{array}{l}\text { (Carolan- } \\
\text { Olah et al, } \\
2018)^{(22)}\end{array}$ & $\begin{array}{l}\text { Communicating out loud: Midwifery } \\
\text { students' experiences of a } \\
\text { simulation exercise for neonatal } \\
\text { resuscitation }\end{array}$ & $\begin{array}{c}\text { Avaliar } \\
\text { cenários de } \\
\text { simulação para } \\
\text { RCP-N }\end{array}$ & $\begin{array}{l}\text { Estudo descritivo, } \\
\text { qualitativo, com } \\
\text { dois cenários de } \\
\text { simulação r para } \\
\text { preparar os } \\
\text { estudantes à } \\
\text { RCP-N. }\end{array}$ & $\begin{array}{l}\text { Os alunos indicaram que } \\
\text { sua confiança e } \\
\text { conhecimento sobre a } \\
\text { RCP } \\
\text { melhoraram. }\end{array}$ & 6 \\
\hline $\begin{array}{l}\text { (León et al, } \\
2015)^{(23)}\end{array}$ & $\begin{array}{l}\text { Comparison of participative } \\
\text { educative strategy versus traditional } \\
\text { educative strategy }\end{array}$ & $\begin{array}{l}\text { Avaliar a } \\
\text { aptidão clínica } \\
\text { para a RCP }\end{array}$ & $\begin{array}{lr}\text { Estudo } & \text { quasi- } \\
\text { experimental, } & \text { com } \\
\text { médicos } & \text { e } \\
\text { enfermeiros. } & \end{array}$ & $\begin{array}{l}\text { Não houve diferenças } \\
\text { entre os grupos na } \\
\text { categoria de avaliação de } \\
\text { aptidão clínica. }\end{array}$ & 6 \\
\hline $\begin{array}{l}\text { (Drake et al, } \\
2019)^{(24)}\end{array}$ & $\begin{array}{l}\text { Structured on-the-job training to } \\
\text { improve retention of newborn } \\
\text { resuscitation skills: a national cohort } \\
\text { Helping Babies Breathe study in } \\
\text { Tanzania }\end{array}$ & $\begin{array}{l}\text { Avaliar a } \\
\text { eficácia de } \\
\text { treinamentos } \\
\text { para RCP-N }\end{array}$ & $\begin{array}{l}\text { Estudo quasi- } \\
\text { experimental } \\
\text { implantação do HBB } \\
\text { na Tanzânia. }\end{array}$ & $\begin{array}{l}\text { Os escores para a } \\
\text { habilidade aumentaram } \\
\text { em ambos os grupos. }\end{array}$ & 3 \\
\hline
\end{tabular}


"continua na página seguinte"

\begin{tabular}{|c|c|c|c|c|c|}
\hline $\begin{array}{c}\text { Autor/ } \\
\text { ano }\end{array}$ & Título do estudo & Objetivo & Método & Resultados/conclusão & NE \\
\hline $\begin{array}{l}\text { (Seto et al, } \\
2017)^{(25)}\end{array}$ & $\begin{array}{l}\text { Breaking Down the Objective } \\
\text { Structured Clinical Examination: An } \\
\text { Evaluation of the Helping Babies } \\
\text { Breathe OSCEs }\end{array}$ & $\begin{array}{l}\text { Avaliar a } \\
\text { eficácia do } \\
\text { OSCE no HBB }\end{array}$ & $\begin{array}{l}\text { Estudo quasi- } \\
\text { experimental, } \\
\text { realizado com } \\
\text { médicos } \\
\text { enfermeiros (70) em } \\
\text { Honduras. }\end{array}$ & $\begin{array}{l}\text { Os escores do pós-teste } \\
\text { foram maiores para } \\
\text { todas as avaliações. }\end{array}$ & 3 \\
\hline
\end{tabular}

NE: nível de evidência; RCP-N: ressuscitação cardiopulmonar neonatal; HBB: Helping Babies Breathe; RCP: ressuscitação cardiopulmonar; UTI: unidade de terapia intensiva; ECOE: Exame Clínico Objetivo Estruturado.

Figura 3 - Delimitação das estratégias educacionais identificadas nos estudos e avaliação de sua efetividade e lacunas quanto ao processo de ensino e aprendizagem da RCP-N.

\begin{tabular}{|c|c|c|}
\hline $\begin{array}{l}\text { Estratégia educacional para o ensino e } \\
\text { aprendizagem da RCP-N }\end{array}$ & $\begin{array}{l}\text { Efetividade da estratégia e lacunas de } \\
\text { ensino e aprendizagem }\end{array}$ & Estudos que utilizaram esta estratégia \\
\hline Programa HBB & $\begin{array}{l}\text { Resultados significativos, para a melhora da } \\
\text { confiança, conhecimento e habilidades para } \\
\text { RCP-N. Diminuição da mortalidade neonatal } \\
\text { significativa nos países em que foi aplicado. } \\
\text { Lacuna para o processo de ensino e } \\
\text { aprendizagem: dificuldade de retenção de } \\
\text { conhecimento em longo prazo após aplicar } \\
\text { esta estratégia. }\end{array}$ & $\begin{array}{l}\text { Kasinea et al }{ }^{(2)} \text {, Perlman et al. }{ }^{(3)} \text {, } \\
\text { Wilson et al. } .^{(3))} \text {, Cordova et al. }{ }^{(17)} \text {, } \\
\text { Ashish et al. }{ }^{(18)} \text {, Drake et al. }{ }^{(24)} \text { e Seto } \\
\text { et al. }{ }^{(25)}\end{array}$ \\
\hline $\begin{array}{l}\text { Simulação realizada em laboratório de } \\
\text { habilidades }\end{array}$ & $\begin{array}{l}\text { Resultados significativos que evidenciam } \\
\text { melhora na confiança, no conhecimento, nas } \\
\text { habilidades, na liderança e no trabalho em } \\
\text { equipe. } \\
\text { Lacuna para o processo de ensino e } \\
\text { aprendizagem: dificuldade de retenção de } \\
\text { conhecimento em longo prazo após aplicar } \\
\text { esta estratégia }\end{array}$ & $\begin{array}{l}\text { Malmström et al. }{ }^{(2)} \text {, Rovamo et al. }{ }^{(9)} \text {, } \\
\text { Saeidi et al. }{ }^{(18)} \text {, Reed et al. }{ }^{(14)} \text {, Carolan- } \\
\text { Olah et al. }{ }^{(21)} \text {, Carolan-Olah et al. }{ }^{(22)}\end{array}$ \\
\hline Simulação in situ & $\begin{array}{l}\text { Resultados estatisticamente significativos } \\
\text { quanto à melhora de conhecimentos e } \\
\text { habilidades para RCP-N. } \\
\text { Lacuna para o processo de ensino e } \\
\text { aprendizagem: dificuldade de retenção de } \\
\text { conhecimento em longo prazo após aplicar } \\
\text { esta estratégia. }\end{array}$ & Brito et al. ${ }^{(8)}$, Vail et al. ${ }^{(19)}$ \\
\hline $\begin{array}{l}\text { Associação de estratégias como: } \\
\text { plataforma on-line de aprendizagem; } \\
\text { cursos presenciais; simulação em } \\
\text { laboratório de habilidades; simulação } \\
\text { in situ; workshops; palestras; } \\
\text { treinamento de habilidades e avaliação } \\
\text { em pares }\end{array}$ & $\begin{array}{l}\text { Resultados estatisticamente significativos } \\
\text { para a melhora na confiança, conhecimentos } \\
\text { e habilidades para RCP-N. } \\
\text { Lacuna para o processo de ensino e } \\
\text { aprendizagem: não foram identificadas. }\end{array}$ & $\begin{array}{l}\text { Shee et al. }{ }^{(11)} \text {, Umar et al. }{ }^{(15)} \text {, } \\
\text { Charafeddine et al. }{ }^{(16)} \text {, Bull et al. }{ }^{(20)} \text {, } \\
\text { León et al. }{ }^{(23)}\end{array}$ \\
\hline
\end{tabular}

RCP-N: ressuscitação cardiopulmonar neonatal; HBB: Helping Babies Breathe.

Os estudos que compuseram a amostra da presente pesquisa são, em sua totalidade, internacionais e dos últimos cinco anos, sendo a maioria de 2017, o que demonstra a atualidade da temática. Grande parte das pesquisas foram classificadas com baixo nível de evidência (nível
6), visto que caracterizaram artigos descritivos, que relataram a abordagem de estratégia educativa para RCP-N $\mathrm{N}^{(3,11-14,16-19,21-22)}$. Identificaram-se, em menor número, estudos quasi-experimentais ${ }^{(2,15-18,20,24-25)}$ e estudos experimentais randomizados ${ }^{(8,9,10)}$, porém apenas 
um estudo experimental ${ }^{(10)}$ demonstrou resultado estatisticamente significativo quanto à efetividade da estratégia educacional escolhida para o ensino da RCP-N. Isso pode justificar a necessidade de explorar este tema, por meio de elaboração de mais estudos experimentais, bem delineados, que apresentem desfechos conclusivos e subsidiem as melhores práticas pedagógicas para o processo de ensino $e$ aprendizagem da RCP neonatal ${ }^{(10)}$.

Embora a adoção de estratégias de ensino apropriadas, para a aprendizagem da RCP-N, não sejam responsáveis exclusivamente pela qualidade deste processo e pela transformação de mecanismos educacionais arcaicos e desconectados com as necessidades dos alunos e de profissionais de enfermagem já inseridos no mercado, investir no conhecimento e exploração de novas possibilidades, pode provocar uma reflexão por parte de instituições de ensino e saúde, em relação às suas práticas e gerar mudanças, permitindo a elaboração de novas concepções pedagógicas que tenham impacto na assistência neonatal ${ }^{(12-14)}$.

Foram elaboradas três categorias quanto aos resultados: (1) principais estratégias de ensino e aprendizagem sobre RCP-N; (2) efetividade das estratégias de ensino e aprendizagem sobre RCP-N; e (3) lacunas no processo de ensino e aprendizagem quanto à estratégia utilizada para RCP-N.

Dentre as estratégias de ensino e aprendizagem sobre RCP-N, identificadas na presente pesquisa, houve destaque para a simulação, seja a realizada em laboratório de habilidades em instituições de ensino ${ }^{(2,9,14,18,21-22)}$ ou in situ ${ }^{(8,19)}$.

Abordagens inovadoras para educação em enfermagem sugerem que o uso de simulação pode ocasionar maior aquisição de conhecimento, confiança e habilidades, sem comprometer a segurança do paciente, porém, embora haja a apresentação de resultados favoráveis à adesão desta estratégia, mais evidências são necessárias, para avaliar a sua eficácia para o contexto neonatal ${ }^{(25)}$.

Para realizar a simulação in situ, isto é, a realização da experiência simulada, no local de trabalho dos profissionais da enfermagem, apontou-se a adoção, principalmente do método, denominado PRONTO ${ }^{(12,19)}$. O método PRONTO é uma abordagem educativa para o ensino da RCP$\mathrm{N}$ in-situ que aborda a aprendizagem em equipe $^{(19)}$.
Estudo transversal de abordagem quantitativa, realizado com 658 enfermeiros em Bihar, na Índia, avaliou o impacto de uma simulação in situ, para ressuscitação neonatal, baseada no método PRONTO, identificando que o processo de ensino e aprendizado realizado, por meio deste método, foi capaz de desenvolver as habilidades essenciais aos profissionais quanto à RCP ${ }^{(19)}$.

A Simulação, dentre as estratégias de ensino para a RCP neonatal, é uma potencial ferramenta de aprendizagem, explorada na atualidade, que pode significar a articulação de teoria e prática e aproximar os educandos e profissionais em enfermagem a cenários mais realistas, que provoquem o pensamento crítico, a reflexão, o trabalho em equipe, representando, desta forma, um meio eficaz para o desenvolvimento de competência clínica quanto à PCR-N ${ }^{(10)}$.

Também houve destaque, para o curso $\mathrm{HBB}^{(2,3,13,17-18,24-25)}$, primeiro módulo de um programa denominado Helping Babies Survive, introduzido, em mais de 80 países, configurando uma estratégia educacional baseada em evidências $^{(36)}$. A maioria dos artigos publicados, em países com poucos recursos, referia-se ao programa HBB, que tem como objetivo ensinar as etapas iniciais da reanimação neonatal ${ }^{(17)}$.

Esta estratégia de ensino para a RCP-N vem ganhando espaço, no contexto mundial e demonstrando-se efetiva, para a aprendizagem de profissionais de enfermagem, principalmente, em contextos em que os recursos humanos e matérias são escassos ${ }^{(13,24-25)}$.

É importante ressaltar que, mesmo diante da importância das estratégias de ensino identificadas para a RCP-N, o processo de aprendizagem, na enfermagem, é complexo e não está somente vinculado aos mecanismos pedagógicos selecionados ${ }^{(21-22)}$.ou ao seu exercício. A aprendizagem de profissionais e estudantes também é influenciada pelo ambiente, pela qualidade do mediador ou professor, recursos materiais e humanos disponíveis, eficácia e qualidade do planejamento educacional, métodos avaliativos propostos, motivação e envolvimento dos participantes, entre outros ${ }^{(17,22-23)}$.

A efetividade das estratégias de ensino e aprendizagem sobre RCP-N foi apresentada como categoria neste estudo e, em geral, todas as estratégias de ensino e aprendizagem identificadas apresentaram-se efetivas, a fim de 
melhorar conhecimentos, habilidades, confiança, liderança e trabalho em equipe, para a ressuscitação neonatal, porém os estudos com desfechos mais conclusivos ${ }^{(11,15-16,20,23)}$ apontam a importância de associar as estratégias de ensino para maximizar o aprendizado. Corroborando com essa afirmação, estudo transversal de abordagem quantitativa sobre ressuscitação neonatal utilizou como estratégia de ensino e aprendizagem a articulação de uma plataforma on-line de aprendizagem, um curso presencial e cenários simulados. Demonstraram-se resultados estatisticamente significativos, para o desenvolvimento de conhecimentos, habilidades e atitudes em RCP-N, pela associação de variadas estratégias pedagógicas ${ }^{(20,23)}$.

As estratégias educacionais, descritas nesta RIL, revelaram como principal lacuna ou fragilidade ao ensino da RCP-N a dificuldade de profissionais e estudantes de enfermagem, em reter o conhecimento, em longo prazo, após a utilização da maioria das estratégias identificadas ${ }^{(2-3,9,13-14,17-22,24-25)}$.

No processo de ensino, para a emergência neonatal, com ênfase na PCR-N, não é cabível considerar que apenas a estratégia utilizada, para este fim, será capaz de provocar retenção de conhecimento e habilidades para enfermagem, visto que já se compreende a importância de se identificar o conhecimento prévio do indivíduo e ancorá-lo aos novos conhecimentos, para que a retenção daquilo que foi aprendido ocorra em longo prazo ${ }^{(23)}$. No entanto a presente pesquisa identificou que a articulação de variadas estratégias de ensino e aprendizagem, para RCP$\mathrm{N}$, pode minimizar essa dificuldade quanto à retenção de conhecimentos, habilidades ou atitudes para $\mathrm{O}$ atendimento PCR-N, indicando como uma alternativa viável ${ }^{(11,15-16,20,23)}$.

\section{CONCLUSÃO}

Os estudos identificados, nesta revisão de literatura, são de cunho internacional, atuais e, em maior parte, de baixo nível de evidência, o que instiga a necessidade de aprofundamento científico e execução de estudos experimentais, randomizados, bem delineados, neste aspecto.

Foram elaboradas três categorias: principais estratégias de ensino e aprendizagem sobre RCP-N; efetividade das estratégias de ensino e aprendizagem sobre RCP-N; e lacunas no processo de ensino e aprendizagem quanto à estratégia utilizada para RCP-N. Dentre as estratégias de ensino e aprendizagem sobre RCP-
$\mathrm{N}$ identificadas estão o programa HBB, a simulação realizada em laboratório de habilidades, simulação in situ e a associação de variadas estratégias pedagógicas, destacando-se a simulação, realizada em laboratório de habilidades ou in situ como a estratégias mais utilizadas para o ensino da RC_neonatal. Todas as estratégias apresentaram-se efetivas quanto ao aperfeiçoamento de conhecimentos, habilidades, confiança, liderança e trabalho em equipe.

A principal lacuna identificada, quanto à aplicação das estratégias pedagógicas evidenciadas, foi a dificuldade em reter conhecimento, o que pode ser melhorado com a associação de variadas estratégias de ensino e aprendizagem para maximizar a habilidade cognitiva dos indivíduos.

A principal limitação deste estudo configurou-se, na falta de clareza quanto ao percurso metodológico adotado pelos estudos e detalhamento da estratégia educacional adotada, o que dificultou sua avaliação.

Este estudo contribui para o ensino, a pesquisa e a assistência, no âmbito da ressuscitação cardiopulmonar neonatal, por identificar as mais atuais estratégias de ensino e aprendizagem adotadas neste âmbito, caracterizar sua efetividade e lacunas, delimitando um arcabouço de conhecimento baseado em evidências para a enfermagem.

\section{REFERÊNCIAS}

1 - Kasinea Y, Babenko-Mouldb Y, Reganc S. Translating continuing professional development education to nursing practice in Rwanda: Enhancing maternal and newborn health. Int J Afr Nurs Sci. 2018;8:75-81. DOI: 10.1016/j.ijans.2018.03.001

2 - Brasil, Ministério da Saúde. Painel de monitoramento da mortalidade infantil e fetal. Brasília: Ministério da Saúde, 2017. [citado em 8 jun 2019]. Disponível em: http://svs.aids.gov.br/dantps/centrais-deconteudos/paineis-demonitoramento/mortalidade/infantil-e-fetal 3 - Perlman J, Msemo G, Ersdal H, Ringia, P. Designing and Implementing the Helping Babies Breathe Program in Tanzania. J Pediatr Intensive Care 2016; 6(1):28-38. DOI: 10.1055/s-00361584674

4 - Mendes KD, Silveira RC, Galvão CM. Revisão integrativa: Método de pesquisa para a incorporação de evidências na saúde e na 
enfermagem. Texto Contexto-Enferm. 2008;17(4):758-64. DOI: $10.1590 / S 0104-$ $\underline{07072008000400018}$

5 - Ouzzani M, Hammady H, Fedorowicz Z, Elmagarmid A. Rayyan - a web and mobile app for systematic reviews. Syst Rev. 2016;5(1):210. DOI: 10.1186/s13643-016-0384-4

6 - Ursi ES, Galvão CM. Prevenção de lesões de pele no perioperatório: revisão integrativa da literatura. Rev. Latino-Am Enfermagem 2006 [citado em 8 jun 2019]; 14(1):124-31. Disponível em:

http://www.scielo.br/pdf/rlae/v14n1/v14n1a17. pdf

7 - Moher D, Liberati A, Tetzlaff J, Altman DG. Preferred reporting items for systematic reviews and metaanalyses: The PRISMA statement. PLoS Med. 2009;6(7):e1000097. DOI: 10.1371/journal.pmed.1000097

8 - Brito JRP, Hughes PG, Firestone KS, Ortiz Figueroa F, Johnson K, Ruthenburg T. Neonatal resuscitation program rolling refresher: Maintaining chest compression proficiency through the use of simulation-based education. Adv Neonatal Care 2017;17(5):354-61. DOI: 10.1097/ANC.0000000000000384

9 - Rovamo L, Nurmi E, Mattila MM, Suominen P, Silvennoinen $M$. Effect of a simulation- based workshop on multidisplinary teamwork of newborn emergencies: An intervention study. BMC Res Notes 2015;8:671. DOI: 10.1186/s13104-015-1654-2

10 - Saeidi R, Gholami M. Comparison of effect of simulation-based neonatal resuscitation education and traditional education on knowledge of nursing students. Iranian Journal of Neonatology. 2017;8(2):1-3. DOI: 10.22038/ijn.2017.23740.1296

11 - Shee A, Gleeson M. Impact of the neoresus training on nonpediatric- trained medical and nursing staff in the emergency department: Experience from two sites in regional Australia. J Clin Neonatol. 2018;7(2):67-70. DOI: 10.4103/jen.JCN 9917

12 - Vail B, Spindler H, Morgan MC, Cohen SR, Christmas A, Sah P, et al. Care of the motherinfant dyad: a novel approach to conducting and evaluating neonatal resuscitation simulation training in Bihar, India. BMC Pregnancy Childbirth 2017;17(1):252. DOI: 10.1186/s12884-017-1434-1 13 - Wilson GM, Ame AM, Khatib MM, Rende EK, Hartman AM, Blood-Siegfried M. Helping babies breathe implementation in Zanzibar, Tanzania.
Int J Nurs Pract. 2017;23(4):e12561. DOI: 10.1111/ijn.12561

14 - Reed DJW, Hermelin RL, Kennedy CS, Sharma J. Interdisciplinary onsite team-based simulation training in the neonatal intensive care unit: a pilot report. J Perinatol. 2017;37(4):461-4. DOI: 10.1038/ip.2016.238

15 - Umar LW, Ahmad HR, Isah A, Idris HW, Hassan L, Abdullahi FL, et al. Evaluation of the cognitive effect of newborn resuscitation training on health-care workers in selected states in Northern Nigeria. Ann Afr Med. 2018;17(1):339. DOI: 10.4103/aam.aam 4717

16 - Charafeddine L, Badran M, Nakad P, Ammar $W$, Yunis K. Strategic assessment of implementation of neonatal resuscitation training at a national level. Pediatr Int. 2016;58(7):595600. DOI: $10.1111 /$ ped.12868

17 - Cordova E, Al-Rousan T, Castillo-Angeles M, Aftab S, Nelson BD. Effect of low-cost interventions on the retention of knowledge and skills following Helping Babies Breathe training. Int J Gynaecol Obstet. 2018;142(2):248-54. DOI: 10.1002/iigo.12512

18 - Ashish KC, Wrammert J, Nelin V, Clark RB, Ewald $U$, Peterson $S$, et al. Evaluation of Helping Babies Breathe Quality Improvement Cycle (HBBQIC) on retention of neonatal resuscitation skills six months after training in Nepal. BMC Pediatr. 2017;17(1):103. DOI: 10.1186/s12887-017-0853-5 19 - Vail B, Morgan MC, Spindler H, Christmas A, Cohen SR, Walker DM. The power of practice: Simulation training improving the quality of neonatal resuscitation skills in Bihar, India. BMC Pediatr. 2018;18(1):291. DOI: 10.1186/s12887$\underline{018-1254-0}$

20 - Bull A, Sweet L. Midwifery students receiving the newborn at birth: A pilot study of the impact of structured training in neonatal resuscitation. Nurse Educ Pract. 2015;15(5):387-92. DOI: 10.1016/j.nepr.2015.03.002

21 - Carolan-Olah M, Kruger G, Brown V, Lawton $F$, Mazzarino M. Development and evaluation of a simulation exercise to prepare midwifery students for neonatal resuscitation. Nurse Educ Today 2016;36:375-80

22 - Carolan-Olah M, Kruger G, Brown V, Lawton F, Mazzarino M, Vasilevski V. Communicating out loud: Midwifery students' experiences of a simulation exercise for neonatal resuscitation. Nurse Educ Pract. 2018;29:8-14. DOI: 10.1016/j.nedt.2015.09.009

23 - León JC, Barajas-Serrano TL, JiménezHernández JE, Barrera-López E, González-Bernal 
C, Higareda-Almaraz MA. [Comparison of participative educative strategy versus traditional educative strategy in health personnel]. Gac Med Mex. 2015 [citado em 8 jun 2019]; 151(3):369-76. Disponível em: http://www.anmm.org.mx/GMM/2015/n3/GMM $\begin{array}{llll}151 & 2015 & 3 & 369-376 . p d f\end{array}$

24 - Drake M, Bishanga DR, Temu A, Njozi $M$, Thomas E, Mponzi V, et al. Structured on-thejob training to improve retention of newborn resuscitation skills: A national cohort Helping Babies Breathe study in Tanzania. BMC Pediatr. 2019;19(1):51. DOI: 10.1186/s12887-019-1419-5 25 - Seto TL, Tabangin ME, Taylor KK, Josyula S, Vasquez JC, Kamath-Rayne BD. Breaking down the objective structured clinical examination: An evaluation of the helping babies breathe osces. Simult Healthc. 2017;12(4):226-32. DOI: $\underline{10.1097 / \mathrm{SIH} .0000000000000224}$

Nota: Não há agência de fomento e não há vínculo com estudo matriz de dissertação, tese, monografia de final de curso ou relatório de pesquisa.

Recebido em: 30/06/2019

Aprovado em: 18/11/2019

Endereço de correspondência: Jordana Luiza Gouvêa de Oliveira Rua: José Minto, número 61. Bairro: Jd. Eldorado. CEP: 14.169-180 - Sertãozinho/SP - Brasil

E-mail: jordana.oliveira@usp.br 